\title{
Elevated Citrate Levels and Histone H3K27M mutations in Pediatric Midline Gliomas-Predictors of Adverse Outcome
}

\author{
Chenue Abongwa ${ }^{1}$, Benita Tamrazi ${ }^{2}$, stefan bluml ${ }^{3}$, Fusheng Yang ${ }^{4}$, Debra Hawes ${ }^{2}$, Nathan \\ Robison $^{5}$, and Girish Dhall ${ }^{6}$ \\ ${ }^{1}$ Children's Hospital Los Angeles Department of Hematology and Oncology \\ ${ }^{2}$ Children's Hospital Los Angeles \\ ${ }^{3}$ USC/Childrens Hospital Los Angeles \\ ${ }^{4}$ Children's Hospital of Los Angeles \\ ${ }^{5}$ Children's Hospital Los Angeles, University of Southern California Keck School of \\ Medicine \\ ${ }^{6}$ University of Alabama at Birmingham
}

November 23, 2020

\begin{abstract}
Background: Low Grade Gliomas(LGG) account for about 50\% of childhood brain tumors. Elevated citrate levels have previously been shown to be markers for aggressive behavior in LGGs and the H3K27M and G34R/V mutations have emerged as molecular drivers for pediatric high-grade gliomas(HGG). Procedure: We retrospectively analyzed data from 25 patients with midline gliomas to determine whether citrate levels measured through magnetic resonance spectroscopy(MRS) were associated with histone mutations identified by immunohistochemistry. Results: Median age was 6 years (range:0-16 years) with 13 males and 12 females. Most of the patients 14(56\%) had a LGG with the most common locations being the thalamus $13(52 \%)$ and brainstem $7(28 \%)$. The majority had undergone a biopsy $16(65 \%)$ or a partial resection $8(32 \%)$. Gross total resection was achieved in only one patient(4\%). The majority of the patients received adjuvant chemotherapy and/or radiotherapy. Median citrate levels trended higher in patients with HGG compared to those with LGG $(1.49 \mathrm{vs} 0.93 \mathrm{mmol} / \mathrm{kg}, \mathrm{p}-\mathrm{value}=0.16)$ and in patients with the H3K27M mutation compared to those without (2.30vs0.80mmol/kg, p-value 0.26). Histological grading (LGGvsHGG) (OR: 3.96, 95\% confidence interval (CI):1.32-11.94), H3K27M mutation (OR:3.77, 95\% CI:1.05-13.61), and age (OR:1.12, 95\%CI:1.00-1.25) were significant adverse prognostic factors in univariate analysis but only the histological grading remained significant in multivariate analysis. Conclusion: We determined that H3K27M mutations and elevated citrate levels were adverse prognostic factors in both patients with LGG and HGG. Exploring the relation between these two markers in larger studies could lead to a noninvasive way of predicting high risk behavior prior to biopsy.
\end{abstract}

\section{INTRODUCTION}

Low Grade Gliomas(LGG) are the most common brain tumor in children accounting for about $50 \%$ of childhood brain tumors(1). A small proportion of LGGs, 2.9\%, have been known to transform to high grade gliomas(HGG)(2) . Genetic markers like the BRAFV600E mutation, have been associated with worse prognosis (3). These molecular studies generally require tissue samples obtained from either a biopsy or resection. Elevated intra-tumoral citrate levels measured noninvasively via magnetic resonance spectroscopy(MRS) has been identified as a marker for those LGG destined to display aggressive behavior(4). The biological basis for the citrate elevation has not yet been fully elucidated but it would appear from initial in vivo studies that the citrate may not be generated at least in the short term from glucose metabolism $(4,5)$.

Recurring mutations with substitution of lysine by methionine at codon 27 in histone H3 variants (H3K27M) 
are found in about $78 \%$ of diffuse intrinsic pontine gliomas (DIPG). In addition, up to $36 \%$ of non-brainstem gliomas carry either the K27M or G34R/V mutations, a mutation characterized by substitution of glycine at codon 34 by arginine or valine. The median survival for these patients is about one year (6-8) (9). Although H3K27M mutations were originally thought to be specific to HGG histology, these mutations were subsequently identified in histological low grade tumors and portended a worse prognosis(10-12). The 2016 WHO Classification of Tumors of the Central Nervous System recently recognized H3K27M mutant diffuse midline glioma as a distinct entity and recommended grading as WHO Grade IV. The mechanism for tumorigenesis for these histone mutations is still being explored but a possible hypothesis is that the H3.3K27M mutation reprograms the epigenetic landscape and gene expression, thus driving tumorigenesis (13).

Determining and understanding the degree to which elevated citrate levels and histone mutations co-occur or occur exclusive of each other could potentially improve the presurgical risk stratification of these patients and identify a molecular pathway which could lead to therapeutic targets. We carried out a retrospective study to determine if higher levels of citrate were associated with histone mutations in pediatric gliomas.

\section{MATERIALS AND METHODS}

\section{Patients}

We included 25 patients, aged 0-21 years with diffuse midline gliomas who had been treated in the Children's Hospital of Los Angeles (CHLA) between September 2000 and December 2012. These patients had citrate levels measured and recorded in an Institutional Review Board (IRB) approved database. Their medical records were reviewed retrospectively for demographic and clinical data including; the date of diagnosis, diagnosis, extent of resection, treatments received, date of progression, cause of death and duration of survival, and data was collected and recorded using a predesigned proforma. Citrate levels and CrammerRao Lower Bounds measurements (CRLB) were obtained from the previously recorded data in the IRB approved database. The CHLA IRB approved the study.

\section{Immunohistochemistry Procedure}

The tissue was fixated in $10 \%$ buffered formalin and embedded in paraffin. The formalin-fixed, paraffinembedded (FFPE) tissue was cut using a microtome into $5 \mu \mathrm{m}$ thick sections, transferred to Superfrost Plus (Shandon Thermo Fisher Scientific, Cheshire, WA) positively charged slides and dried at $70^{\circ} \mathrm{C}$ for 30 minutes. Immunohistochemistry (IHC) for H3K27M was performed using the Leica Bond RX Autostainer (Leica Biosystems, Buffalo Grove, IL) and the Bond Rx. The slides were deparaffinized and antigen retrieval was performed in the Bond Rx system with Epitope Retrieval 1 solution ( $\mathrm{pH} 6$ ) for 30 minutes. The sections were then incubated for 30 minutes with rabbit polyclonal anti-H3K27M (Millipore, MAB419, Burlington, MA) at a concentration of 1:600 for 30 minutes using the standard Leica Bond protocol IHC-F. The Leica DAB detection kit (Leica Biosystems, Buffalo Grove, IL) was used according to the manufacturer's instructions and the slides were counter-stained with hematoxylin and cover slipped using the Thermo ClearVue automated cover slipper (Thero Fisher, Kalamazoo, MI).

The slides were read by a board-certified pediatric neuropathologist. A slide was determined to be positive if there was nuclear expression of the antibody in the tumor cells and negative if there was no expression in the tumor cells in the presence of adequate positive and negative controls. H3K27M mutation generally shows global expression of the marker by IHC. Cases with rare positive cells or equivocal staining were considered negative.

\section{Citrate Level Measurement}

Proton MRS were acquired using a single-voxel point-resolved spectroscopy (PRESS) with a short echo time (TE) of 35 milliseconds, a repetition time (TR) of 1.5 seconds, and 128 signal averages were used for all acquisitions(14). The total acquisition time, which included scanner adjustments, was less than five minutes per spectrum. All spectra were processed with fully automated LCModel (Stephen Provencher Inc., Oakville, Ontario, Canada, LCModel Version 6.3-1L) software and citrate was quantified as previously 
described $(4,15)$. MRS data was acquired prior to contrast injections using a 1.5T MR System (Sign LX; EG Healthcare). Patients aged less than 5 years were imaged under general anesthesia. Single voxel short TE was used to obtain signals with minimal signal loss due to T2 signal decay of metabolites. T2-weighed fast spin-echo, fluid-attenuated inversion recovery (FLAIR), and T1-weighed FLAIR magnetic resonance (MR) images were acquired in all cases and reviewed to determine the extent of the lesion prior to selecting the region of interest. This region was documented in three MRIs and reviewed to ensure that no partial volume for normal appearing tissue was enclosed. Sizes and shaped of this region were adjusted to the lesion sizes and typically varied from $5-10 \mathrm{~cm}^{3}$ (Figure 1$)$.

\section{Statistical Analysis}

Descriptive statistics were used to summarize the distribution of each variable and to compare the baseline characteristics of patients. The chi-square and/or Fisher's exact tests were used to compare differences in categorical variables between patients. The Student t-test and Wilcoxon rank sum nonparametric statistics were used for similar comparisons with continuous variables. All p-values were two tailed with p-values $<0.05$ considered significant. The Kaplan-Meier (KM) method was used to estimate unadjusted overall survival (OS) probabilities. The log-rank test was used to compare differences in OS between patients with low grade glioma, high grade glioma, those with or without H3K27M mutations and those with or without elevations in citrate levels. Median follow-up time was determined using the reverse KM method. All data analyzed using and R statistical software (R Foundation for Statistical Computing, Vienna, Austria).

\section{RESULTS}

\section{General Characteristics}

The median age of the patients was 6-years (range: 0-16 years) and there were 13 males (52\%) and 12 females (48\%). The majority of the patients had low-grade gliomas, 14 vs 11 (56 vs $44 \%$ ), and the tumors were predominantly located in the thalamic region $13(52 \%)$, basal ganglia $4(16 \%)$, brainstem $7(28 \%)$ and optic chiasma/hypothalamic region 1 (4\%). The majority of the patients had undergone biopsies $16(65 \%)$, and partial resections $8(32 \%)$. Gross total resection was only achieved in one patient (4\%). Most of the patients were treated subsequently with adjuvant chemotherapy and radiotherapy $8(36.4 \%)$. Eight patients $(36.4 \%)$ underwent further surgery in addition to chemotherapy, and radiotherapy during the course of their disease. Fifteen patients (60\%) ultimately died of their disease (Table 1).

\section{H3K27M Mutation and Citrate Levels}

Of the 25 patients included, H3K27M mutations were identified in $7(28 \%)$ patients, while $14(56 \%)$ patients had the wild type phenotype. Four of the patients (16\%) despite having a histologic diagnosis, did not have sufficient tissue to perform the H3K27M mutation studies and were excluded in the subsequent analysis. The median citrate level trended higher in patients with HGG when compared to those with LGG (1.49 vs $0.93 \mathrm{mmol} / \mathrm{kg}$, p-value $=0.16)$ and in the seven patients $(33 \%)$ with H3K27M mutation when compared to those without (2.25 vs $0.78 \mathrm{mmol} / \mathrm{kg}$, p-value 0.25$)$ but this was not statistically significant (Table 2$)$. The majority of patients with H3K27M mutation had HGGs by histological grading (6/7) with only one patient having a LGG.

On univariate analysis, the tumor histological grade (OR: 3.96, 95\% confidence interval (CI): 1.32-11.94), H3K27M mutation (OR: 3.77, 95\% CI: 1.05-13.61) and age (OR:1.12, 95\% CI: 1.00-1.25) were statistically significant predictors for adverse prognosis (Table 3). Elevated citrate concentration $>1.2 \mathrm{mmol} / \mathrm{kg}$ tended to predict adverse prognosis, but this was not statistically significant (OR: 2.92, 95\% CI: 0.91-2.23). The median duration of follow up was 113 months (range: 7-209 months) and the median OS for all patients was 67 months (range: 4-138 months). The median survival was significantly lower in patients with H3K27M mutations when compared to those without (24 vs 138 months, p value $<0.05$ ), in patients with elevated citrate levels $>1.2 \mathrm{mmol} / \mathrm{kg}$ when compared to those without (24 vs 105 months, p-value 0.07 ) and in patients with HGG when compared to LGG (16 vs 138 months, p value 0.012). On multivariate analysis only the histological grade (HGG vs LGG) was statistically significant (OR: 6.02, 95\% CI:1.05-34.64, p-value=0.04) 
(Table 3, Figure 2).

\section{DISCUSSION}

This is one of the first pediatric studies comparing citrate levels in patients with midline glioma with or without the H3K27M mutation. Histone mutations have recently emerged as important molecular drivers and poor markers for pediatric brain tumors. The H3K27M midline gliomas have been included in the recent 2016 WHO classification of tumors of the central nervous system as a separate entity(16). Prior studies have also reported on elevated citrate levels in low-grade gliomas destined for aggressive behavior(4). Our study shows some interesting findings. Patients with the H3K27M mutation demonstrated higher citrate levels when compared to patients the mutation and the expression did not seem to be dependent on the histological grade of the tumor. Patients with H3K27M the mutation had a statistically significant worse median OS when compared those without. Patients with elevated citrate levels $>1.2 \mathrm{mmol} / \mathrm{kg}$ tended to have a worse prognosis, but this was not statistically significant.

Similar findings have been reported in other studies. Bluml et al reported on elevated mean citrate levels in patients with aggressive when compared to indolent low-grade glioma $(4.1 \pm 1.1$ vs $0.6 \pm 0.8 \mathrm{mmol} / \mathrm{kg}$; $\mathrm{p}<0.01)(4)$. Their citrate level measurements, however, were inconsistent in the high-grade glioma patients with some of these patients demonstrating elevated citrate levels and others not (4). A similar finding was noted in our study with the range varying from $0-7.5 \mathrm{nmol} / \mathrm{g}$, but the median citrate level was significantly higher for the histologically WHO Grade III/IV when compared to the WHO Grade I/II patients. The majority of patients with the H3K27M mutation had grade III or IV tumors by histology (6/7), similar to other prior reports (10). One of the patients with a histologic low grade glioma also carried this mutation, supporting other published reports $(11,12)$. The prognosis of the patients with the H3K27M mutation in our study was also poor, consistent with other studies(6, 17-19). H3K27M mutant diffuse midline gliomas are now considered WHO Grade IV irrespective of the presence of anaplastic features in the 2016 WHO classification of tumors of the CNS because of this association with a poor prognosis (16).

The mechanism through which H3K27M and other histone mutations could alter citrate metabolism has not yet been studied. H3K27 is a substrate of the polycomb repressive complex 2 (PRC2), a major regulator of gene expression (20). PRC2 silences gene expression through trimethylation of K27 of the histone $\mathrm{H} 3(\mathrm{H} 3 \mathrm{~K} 27 \mathrm{me} 3)$ via its catalytic SET domain. H3K27M is a missense mutation in H3K27 and is associated with global decrease in H3K27me3, which is thought to be mediated through inhibition of PRC2 activity (20). These mutations are involved in oncogenesis of pediatric cancers but could in addition, be associated with alterations in the expression of other genes, leading to changes in downstream metabolic pathways, including that of citrate metabolism, thus leading to elevated citrate levels $(5,6)(13)$. More work obviously is required to fully understand these pathways. The finding that citrate is elevated in our study, if confirmed in larger studies could potentially suggest cellular pathways that could be explored to understand the metabolic alterations in these tumors.

Our study has a few limitations. The small sample size made it impossible to explore some of the relationships involving these variables in depth and suggest some of these findings could have occurred by chance. Although the histological grade, H3K27M mutations and age independently predicted a poor prognosis in univariate analysis, the finding that only the histological grading was statistically significantly associated with a poor outcome in multivariate analysis, suggests the sample size may have been too small to detect a change in the other variables. Although it would have been of interest to study how the H3K27M mutation affected the prognosis in histologically WHO Grade I/II and WHO Grade III/IV subgroups, this was not possible because of the small number of patients. Both a higher histological grading (WHO III/IV) and H3K27M mutation portended a worse prognosis in our report supporting the integrating of histologically and molecular data in diagnosing these tumors as in the 2016 WHO classification (21).

Our study, nonetheless, provides important hypotheses which could be tested in future studies, such as, exploring the relationship between the H3K27M mutation and citrate metabolism in a larger study to further understand the relationship between these two prognostic markers, and the utility of citrate levels 
preoperatively in consistently identifying a subgroup of more aggressive tumors histologically. In addition, with MR spectroscopy now more readily available in many different centers, this could improve the preoperative characterization of tumors.

\section{REFERENCES:}

1. Qaddoumi I, Sultan I, Broniscer A. Pediatric low-grade gliomas and the need for new options for therapy: Why and how? Cancer Biol Ther. 2009;8(1):4-10.

2. Mistry M, Zhukova N, Merico D, Rakopoulos P, Krishnatry R, Shago M, et al. BRAF mutation and CDKN2A deletion define a clinically distinct subgroup of childhood secondary high-grade glioma. $J$ Clin Oncol.2015 Mar 20;33(9):1015-22.

3. Lassaletta A, Zapotocky M, Mistry M, Ramaswamy V, Honnorat M, Krishnatry R, et al. Therapeutic and Prognostic Implications of BRAF V600E in Pediatric Low-Grade Gliomas. J Clin Oncol. 2017 Sep 1;35(25):2934-2941.

4. Bluml S, Panigrahy A, Laskov M, Dhall G, Krieger MD, Nelson MD, et al. Elevated citrate in pediatric astrocytomas with malignant progression. Neuro Oncol. 2011;13(10):1107-17.

5. Choi C, Ganji SK, Madan A, Hulsey KM, An Z, Zhang S, et al. In vivo detection of citrate in brain tumors by 1H magnetic resonance spectroscopy at 3T. Magn Reson Med. 2014;72(2):316-23.

6. Sturm D, Witt H, Hovestadt V, Khuong-Quang DA, Jones DT, Konermann C, et al. Hotspot mutations in H3F3A and IDH1 define distinct epigenetic and biological subgroups of glioblastoma. Cancer Cell.2012;22(4):425-37.

7. Schwartzentruber J, Korshunov A, Liu XY, Jones DT, Pfaff E, Jacob K, et al. Driver mutations in histone H3.3 and chromatin remodelling genes in paediatric glioblastoma. Nature. 2012;482(7384):226-31.

8. Wu G, Broniscer A, McEachron TA, Lu C, Paugh BS, Becksfort J, et al. Somatic histone H3 alterations in pediatric diffuse intrinsic pontine gliomas and non-brainstem glioblastomas. Nat Genet.2012;44(3):251-3.

9. Kallappagoudar S, Yadav RK, Lowe BR, Partridge JF. Histone H3 mutations-a special role for H3.3 in tumorigenesis? Chromosoma . 2015;124(2):177-89.

10. Gielen GH, Gessi M, Hammes J, Kramm CM, Waha A, Pietsch T. H3F3A K27M mutation in pediatric CNS tumors: a marker for diffuse high-grade astrocytomas. Am J Clin Pathol. 2013;139(3):345-9.

11. Hochart A, Escande F, Rocourt N, Grill J, Koubi-Pick V, Beaujot J, et al. Long survival in a child with a mutated K27M-H3.3 pilocytic astrocytoma. Ann Clin Transl Neurol . 2015;2(4):439-43.

12. Orillac C, Thomas C, Dastagirzada Y, Hidalgo ET, Golfinos JG, Zagzag D, et al. Pilocytic astrocytoma and glioneuronal tumor with histone H3 K27M mutation. Acta Neuropathol Commun . 2016;4(1):84.

13. Chan KM, Fang D, Gan H, Hashizume R, Yu C, Schroeder M, et al. The histone H3.3K27M mutation in pediatric glioma reprograms H3K27 methylation and gene expression. Genes Dev . 2013;27(9):985-90.

14. Bottomley PA, Foster TH, Leue WM. Chemical imaging of the brain by NMR. Lancet . 1984;1(8386):1120.

15. Provencher SW. Estimation of metabolite concentrations from localized in vivo proton NMR spectra. Magn Reson Med.1993;30(6):672-9.

16. Louis DN, Perry A, Reifenberger G, von Deimling A, Figarella-Branger D, Cavenee WK, et al. The 2016 World Health Organization Classification of Tumors of the Central Nervous System: a summary. Acta Neuropathol. 2016;131(6):803-20.

17. Buczkowicz P, Bartels U, Bouffet E, Becher O, Hawkins C. Histopathological spectrum of paediatric diffuse intrinsic pontine glioma: diagnostic and therapeutic implications. Acta Neuropathol. 2014;128(4):57381. 
18. Gessi M, Gielen GH, Dreschmann V, Waha A, Pietsch T. High frequency of H3F3A (K27M) mutations characterizes pediatric and adult high-grade gliomas of the spinal cord. Acta Neuropathol . 2015;130(3):435-7.

19. Korshunov A, Ryzhova M, Hovestadt V, Bender S, Sturm D, Capper D, et al. Integrated analysis of pediatric glioblastoma reveals a subset of biologically favorable tumors with associated molecular prognostic markers. Acta Neuropathol . 2015;129(5):669-78.

20. Justin N, Zhang Y, Tarricone C, Martin SR, Chen S, Underwood E, et al. Structural basis of oncogenic histone H3K27M inhibition of human polycomb repressive complex 2. Nat Commun. 2016;7:11316.

21. Pages M, Beccaria K, Boddaert N, Saffroy R, Besnard A, Castel D, et al. Co-occurrence of histone H3 K27M and BRAF V600E mutations in paediatric midline grade I ganglioglioma. Brain Pathol.2018;28(1):10311.

Figure 1. a. Median overall survival was 67 months. b. Patients with H3K27M mutations had a shorter overall survival c. Patients with citrate levels $>1.2$ had a shorter overall survival but this was not significant. d. Patients with high grade gliomas had a shorter survival as expected.

Figure 2. In vivo MR spectrum acquired with a point-resolved spectroscopy (PRESS) sequence with an echo time of $35 \mathrm{~ms}$ of a bi-thalamic astrocytoma at initial presentation. Citrate (Cit) is detectable at approximately $2.6 \mathrm{ppm}$. Other chemicals that can be observed with in vivo MRS not analyzed for this report) include choline (Cho), creatine (Cr), N-acetyl-aspartate (NAA), myo-inositol (mI), and glycine (Glyc, overlapping with $\mathrm{mI}$ ). Subsequent to the MR examination a biopsy was performed, and a diffuse astrocytoma (WHO II) was diagnosed. The tumor, however, progressed rapidly within 6 months.

\section{Hosted file}

Table 1.pdf available at https://authorea.com/users/377922/articles/494546-elevated-citratelevels-and-histone-h3k27m-mutations-in-pediatric-midline-gliomas-predictors-of-adverseoutcome

\section{Hosted file}

Table 2.pdf available at https://authorea.com/users/377922/articles/494546-elevated-citratelevels-and-histone-h3k27m-mutations-in-pediatric-midline-gliomas-predictors-of-adverseoutcome

\section{Hosted file}

Table 3.pdf available at https://authorea.com/users/377922/articles/494546-elevated-citratelevels-and-histone-h3k27m-mutations-in-pediatric-midline-gliomas-predictors-of-adverseoutcome 

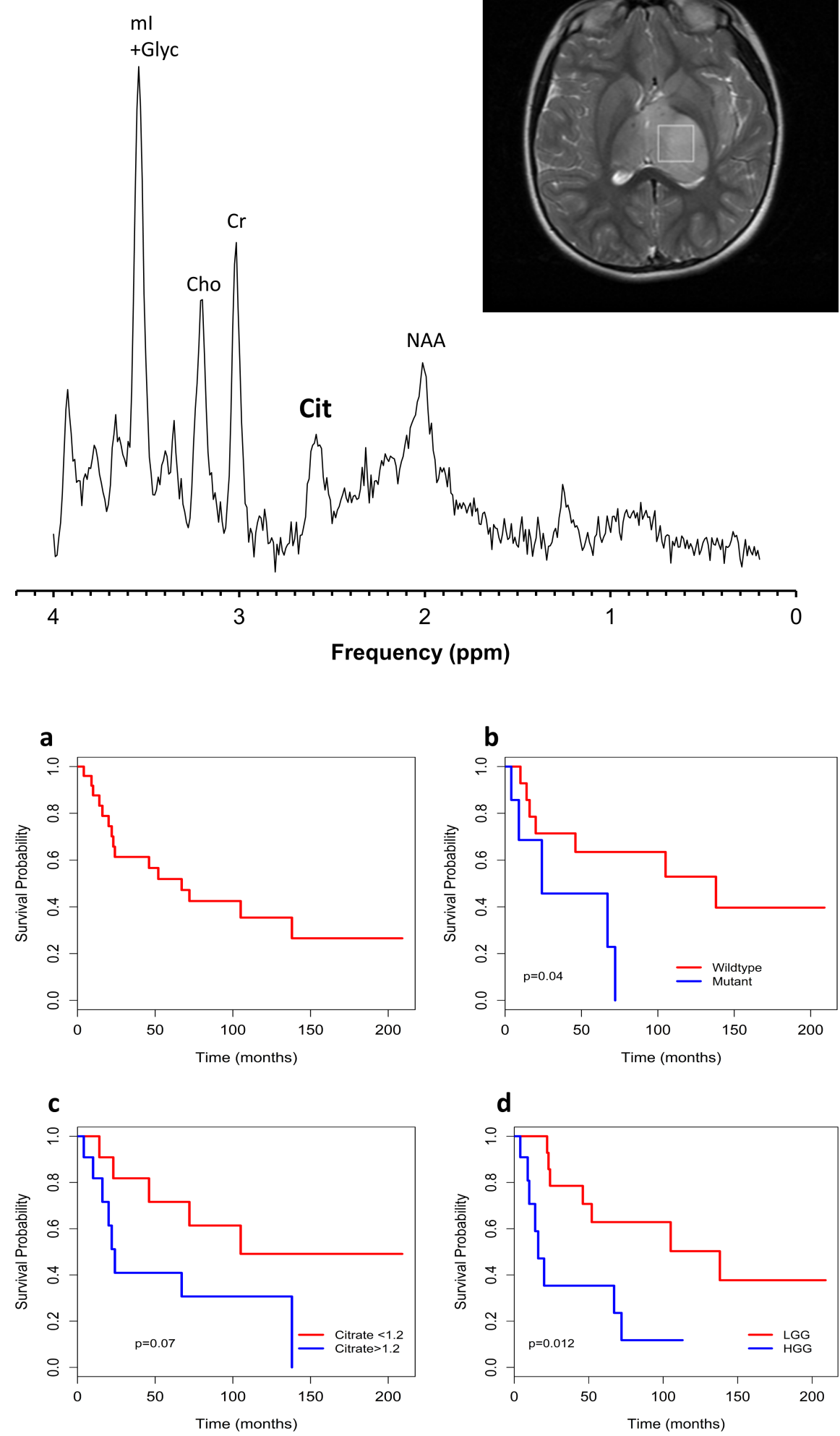